\title{
PREPARATION OF CROSS-SECTIONAL TEM SPECIMENS OF OBLIQUELY DEPOSITED MAGNETIC THIN FILMS ON A FLEXIBLE TAPE; ELECTRON TRANSPARENCY BEYOND 6 MICRON !
}

\author{
Enrico G. Keim*, L.T. Nguyen ${ }^{* *}$ and J. Cock Lodder ${ }^{* *}$ \\ ${ }^{*} \mathrm{MESA}^{+}$Research Institute, Central Materials Analysis Laboratory, University of Twente, P.O. Box \\ 217, 7500 AE Enschede, The Netherlands \\ ${ }^{* *} \mathrm{MESA}^{+}$Research Institute, Systems and Materials for Information storage (SMI), University of \\ Twente, P.O. Box 217, 7500 AE Enschede, The Netherlands.
}

Transmission Electron Microscopy (TEM) cross-sections of metal sputtered or metal evaporated tapes for magnetic recording pose several unique problems during preparation. An important property of such systems is the fact that we have to deal with a ductile, fragile metal layer on top of a tough polymer. The latter is typically of $10 \mu \mathrm{m}$ thickness, while the layer of interest is in the $20-200 \mathrm{~nm}$ range. One of the most challenging aspects of such a preparation procedure is to create a homogeneous electron transparent region which is as large as possible, and leaving metal film, its substrate and glue layer intact. In our recent publication [1] we have shown that the preparation of such cross-sections for TEM observation, using standard tools like a dimple grinder and a precision ion etching system, leads to essentially artifact-free and relatively large homogeneous electron transparency in the order of 2 microns along the interface (see Fig. 1b). The only draw-back being however, the long preparation time to accomplish such cross-sections, which is mainly due to the use of a two-component resin that cures at room temperature (see Ref. [1]). In the present work we will show the results of TEM observations on cross-sections of thin obliquely deposited metal double-layer films on a polymer substrate (PET). Essential differences with the previously described recipe [1] are the application of an extremely small amount of fast-curing two-component resin (GATAN-G1), and the use of only one metal doublelayer/PET sample in the "sandwich".

Oblique deposition of magnetic films is often used for magnetic recording tape. Widely used materials are $\mathrm{Co}$ and $\mathrm{CoNi}$ which are deposited directly onto PET by reactive evaporation with $\mathrm{O}_{2}[2]$. However, for the next generation of high density media the appropriate desirable magnetic properties cannot be realized by this method [3]. Therefore, a detailed investigation into the effect of a $\mathrm{Cr}$ underlayer on obliquely sputtered Co on a PET substrate at an incident angle of 70 degrees has been undertaken. The results of the magnetic analyses will be reported elsewhere [4]. The microstructure and magnetic analyses of the Co layer reveal a strong influence of the $\mathrm{Cr}$ underlayer. With a Cr underlayer the Co films consist mainly of the hcp crystalline structure (electron diffraction micrographs not shown here, see Ref. 4). As a result of the oblique incident condition, tilted columns have, as expected, been observed in the case of thick Co and Cr layers as shown in Fig.1a in which single metal columns can be observed unambiguously. For comparison, we also show a TEM image in cross-section of a metal sputtered Co film on PET (Fig. 1b) published in Ref. [1].

It has been demonstrated that refinements of our previous recipe [1] yield reproducible artifact-free cross-sectional TEM specimens showing homogeneous electron transparent regions exceeding $6 \mu \mathrm{m}$. Moreover, using the same standard fast-preparation tools [1] we can now routinely prepare such crosssections and perform TEM analysis on these within one day. 


\section{References}

[1] E.G. Keim et al., J. Vac. Sci. Technol. A19 (2001) 1191.

[2] L. Abelmann and J.C. Lodder, Thin Solid Films 305 (1997) 1.

[3] A. Lisfi and J.C. Lodder, Phys. Rev. B63, (2001) 174441.

[4] L.T. Nguyen et al., presentation at Intermag Europe 2002, April $28^{\text {th }}-$ May $2^{\text {nd }} 2002$, Amsterdam, The Netherlands.

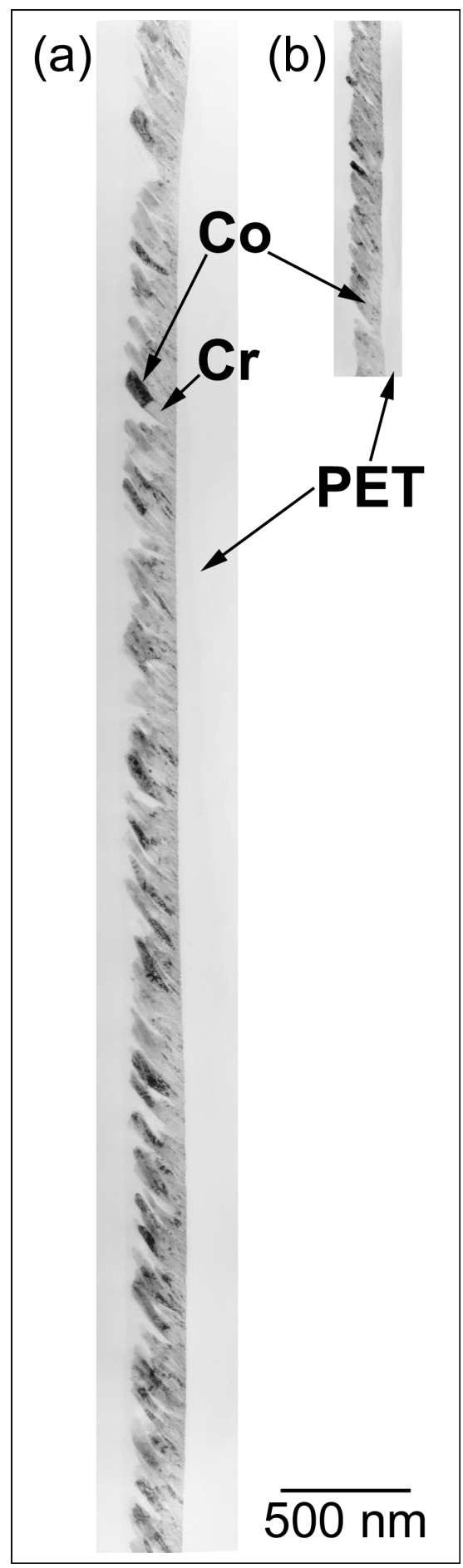

FIG. 1 (a) Low-magnification cross-sectional TEM image of an obliquely deposited metal double-layer film, consisting of $120 \mathrm{~nm}$ thick sputtered Co on a $120 \mathrm{~nm}$ thick Cr underlayer; (b) shows a cross-sectional TEM image of a metal sputtered Co film on PET as prepared using our previous recipe [1]. The scale bar in this figure represents both (a) and (b). 